Research Paper

\title{
Matrigel Scaffolding Enhances BMP9-induced Bone Formation in Dental Follicle Stem/Precursor Cells
}

Tiwei Fu ${ }^{1,2^{*}}$, Panpan Liang ${ }^{1,2^{*}}$, Jinlin Song1,2, Jinhua Wang1,2, Pengfei Zhou ${ }^{1,2}$, Yinhong Tang ${ }^{1,2}$, Jing Li ${ }^{1,2}$, Enyi Huang $1,2 \llbracket$

1. Chongqing Key Laboratory of Oral Diseases and Biomedical Sciences, Stomatological Hospital of Chongqing Medical University, Chongqing 401147, P.R. China

2. Chongqing Municipal Key Laboratory of Oral Biomedical Engineering of Higher Education College of Stomatology, College of Stomatology, Chongqing Medical University, Chongqing 400016, P.R. China

*These authors contributed equally to the work.

$\triangle$ Corresponding author: Enyi Huang, Phone: 86-23-88602356; Email: enyihuang@hospital.cqmu.edu.cn

(C) Ivyspring International Publisher. This is an open access article distributed under the terms of the Creative Commons Attribution (CC BY-NC) license (https://creativecommons.org/licenses/by-nc/4.0/). See http://ivyspring.com/terms for full terms and conditions.

Received: 2018.10.19; Accepted: 2019.03.21; Published: 2019.04.25

\begin{abstract}
Bone tissue engineering requires a combination of cells, efficient biochemical and physicochemical factors, and biocompatible scaffolds. In this study, we evaluated the potential use of injectable Matrigel as a scaffold for the delivery of rat dental follicle stem/precursor cells (rDFSCs) transduced by bone morphogenetic protein (BMP) 9 to enhance osteogenic differentiation in vitro and promote ectopic bone formation in vivo. Recombinant adenovirus was used to overexpress BMP9 in rDFSCs. Alkaline phosphatase activity was measured using a histochemical staining assay and a chemiluminescence assay kit. Quantitative real-time polymerase chain reaction was used to determine mRNA expression levels of bone-related genes including distal-less homeobox 5 (DLX5), osteopontin (OPN), osterix (Osx), and runt-related transcription factor 2 (Runx2). Matrix mineralization was examined by Alizarin Red S staining. rDFSCs proliferation was analyzed using the Cell Counting Kit-8 assay. Subcutaneous implantation of rDFSCs-containing Matrigel scaffolds was used, and micro-computed tomography analysis, histological evaluation, and trichrome staining of implants extracted at 6 weeks were performed. We found that BMP9 enhanced alkaline phosphatase activity and mineralization in rDFSCs. The expression of bone-related genes (DLX5, OPN, Osx, and Runx2) was also increased as a result of BMP9 stimulation. Micro-computed tomography analysis and histological evaluation revealed that the bone masses retrieved from BMP9-overexpressing rDFSCs were significantly more pronounced in those with than in those without Matrigel. Our results suggest that BMP9 effectively promote osteogenic differentiation of rDFSCs, and Matrigel facilitate BMP9-induced osteogenesis of rDFSCs in vivo.
\end{abstract}

Key words: matrigel, BMP9, rDFSCs, bone formation

\section{Introduction}

Bone defects caused by trauma, tumors, or other pathological events are major clinical and socioeconomic burdens [1]. Currently, the gold standard for treatment of patients suffering from bone defects is to perform bone grafting, using either an autograft or an allograft $[2,3]$. However, surgical grafting for autografts causes complications, including new wounds on the donor site, morbidity at the harvest site, and local hematoma and remodeling issues of the implanted bone, which induce secondary trauma and pain $[4,5]$. The allograft is hampered by bone tissue integration from the host and vascularization tissues [3]. Bone tissue engineering is a rapidly developing strategy for repairing critically sized bone defects to address the need for bone augmentation and skeletal repair [6]. Overall, an effective approach to bone tissue engineering aims to regenerate damaged bone tissue by combining three key components: growth factors, functional cells, and biomaterial scaffolds.

Growth factors, which are essential for cellular proliferation, differentiation, and maturation, are important components in bone tissue engineering [7]. Bone morphogenetic proteins (BMPs) are a family of pleiotropic morphogens that belong to the transforming growth factor- $\beta$ superfamily and play an essential role in regulating embryonic and postnatal 
development, as well as in the formation of skeletal tissues [8]. There are at least 14 different BMPs in humans, and BMP2, BMP4, BMP6, BMP7, and BMP9 play important roles in inducing osteoblast differentiation of mesenchymal stem cells (MSCs) [9]. Among these, BMP2 and BMP7 have been approved by the Food and Drug Administration for the treatment of spine fusions, tibia fractures, and bone defects $[8,10]$. Despite the widespread use of both BMP2 and BMP7, we are interested in BMP9 because it is one of the most osteogenic yet least studied BMPs. Luu et al and Kang et al conducted a comprehensive analysis of the 14 types of BMPs for their abilities to regulate multilineage specific differentiation of MSCs. They found that BMP2, 7, and 9 demonstrated varying degrees of ossification in stem cell transplantation experiments. BMP2 and 7 were the least developed with small foci of woven bone. However, BMP9 had multiple foci of immature woven trabecular bone. Treating MSCs with BMP3, a known negative regulator of bone formation, BMP2-induced ossification was completely blocked, and most of the BMP7induced ossification was inhibited by BMP3. However, BMP3 did not exert any effect on BMP9induced ossification [9, 11]. BMP9 was originally identified in fetal mouse liver. The function of BMP9 is not only to induce osteogenic differentiation, but also to induce and maintain cholinergic phenotype of basal forebrain neurons, induce the expression of critical enzymes of lipid metabolism, stimulat hepcidin 1 expression, regulate angiogenesis and inhibit hepatic glucose production [12]. Recent reports have demonstrated that BMP9 signaling has extensive cross-talks with other signaling pathways, especially Wnt and Notch signaling [12-14].

Dental follicle stem/precursor cells (DFSCs) are a type of dental MSCs identified from dental follicle tissues, and these cells have been shown to differentiate into osteoblasts/cementoblasts, adipocytes, and neurons $[15,16]$. DFSCs are easily isolated and readily grow under culture conditions, which have important potential applications in bone tissue engineering [16]. The development of a suitable scaffolding system is pivotal in engineering tissue regeneration. The ideal scaffold should fill irregular- shaped defects, be easy to handle, and promote bone formation.

Three-dimensional (3D) scaffolds are typically porous, biocompatible and biodegradable materials that serve to provide structural support for cells and the new tissue being formed, acting as a temporary extracellular matrix inducing the natural processes of tissue regeneration and development[17, 18]. Matrigel is a soluble and sterile extract of basement membrane proteins derived from Englebreth-Holm-Swarm tumors in mice, which exists in the liquid forms at $4^{\circ} \mathrm{C}$ but forms a porous clear gel at $37^{\circ} \mathrm{C}$ [19]. Matrigel has been widely investigated in tissue engineering to serve as an injectable scaffold. It was reported that VEGF-Loaded PEG/PCL-Based hydrogel scaffold coating with Matrigel promoted the angiogenesis when Bone-marrow derived mesenchymal stem cells (hBMSCs) were transplanted in BALB/c nude mice [20]. Matrigel mixed with bovine mammary epithelial cells formed bovine mammary gland in the dorsal tissue of nude mice [21]. Previous studies of Matrigel have focused on its application in the regeneration of soft tissue. However, information about the effects of Matrigel on hard tissue is limited. Therefore, in the present study, we used Matrigel as a delivery scaffold for BMP9-induced rat DFSCs (rDFSCs) to construct xenogeneic biological scaffolds for bone formation.

\section{Materials and methods}

\section{Cell culture and adenoviral infection}

Rat DFSCs were obtained and cultured as described previously [22]. Briefly, dental follicles were obtained from the first mandibular molars of rat pups at days 5-7 postnatally and digested with type I collagenase and trypsin. The cells were then maintained in complete a-minimal essential medium (a-MEM; Hyclone, Logan, UT, USA) supplemented with $10 \%$ fetal calf serum (Hyclone), $100 \mathrm{U} / \mathrm{mL}$ penicillin, and $100 \mathrm{~g} / \mathrm{mL}$ streptomycin (Beyotime, Shanghai, China) in a humidified $37^{\circ} \mathrm{C}$ incubator with $5 \% \mathrm{CO}_{2}$. Recombinant adenovirus-expressing human BMP9 (Ad-BMP9) and green fluorescent protein (Ad-GFP) were constructed using the AdEasy system as described previously [23]. Cells were passaged to $60 \%$ confluence and then infected with Ad-BMP9 or Ad-GFP. Polybrene was added together with the virus at a concentration of $1 \mu \mathrm{L} / \mathrm{mL}$ in medium to increase infection efficiency.

\section{Alkaline phosphatase (ALP) activity assay}

ALP activity and staining were assessed as described previously [23, 24]. The rDFSCs were seeded into 24-well plates and transduced with BMP9 or GFP. ALP activity was measured quantitatively by the Great Escape SEAP Chemiluminescence Assay Kit (BD Clontech, Palo Alto, CA, USA) and qualitatively by a histochemical staining assay using a mixture of $0.1 \mathrm{mg} / \mathrm{mL}$ naphthol AS-MX phosphate and 0.6 $\mathrm{mg} / \mathrm{mL}$ Fast Blue BB Salt (Sigma-Aldrich, St. Louis, MO, USA) on days 3 and 5. ALP activity was normalized to the total cellular protein concentration.

\section{Alizarin Red S staining}

Ad-BMP9- or Ad-GFP-infected cells were cultured in the presence of $50 \mathrm{mg} / \mathrm{mL}$ ascorbic acid (Sigma-Aldrich) and $10 \mathrm{mM} \beta$-glycerophosphate 
(Sigma-Aldrich). On day 14 of culture, the cells were harvested for Alizarin Red S staining (Sigma-Aldrich) to determine bone matrix mineralization, as described previously [23, 24]. The cells were washed with phosphate-buffered saline (PBS) twice and fixed with $0.05 \%(\mathrm{v} / \mathrm{v})$ glutaraldehyde for $10 \mathrm{~min}$ at room temperature. After washing with double-distilled water, the cells were stained with $2 \%$ Alizarin Red S at $37^{\circ} \mathrm{C}$ for $20 \mathrm{~min}$. The results were recorded using a bright field microscope.

\section{Quantitative real-time polymerase chain reaction (qRT-PCR)}

Total RNA was extracted using TRIzol reagent (Takara, Dalian, China) and $1 \mu \mathrm{g}$ total RNA was reverse transcribed into $\mathrm{cDNA}$ by using the PrimeScript RT Master Mix cDNA Synthesis Kit (Takara). All reactions were performed on the CFX96 Real-Time PCR Detection System (Bio-Rad, Hercules, CA, USA). Target gene expression was normalized to that of glyceraldehyde 3-phosphate dehydrogenase (GAPDH). The following qRT-PCR primer sequences were designed using Primer3 Plus (http://www. bioinformatics.nl/cgi-bin/primer3plus/primer3plus. cgi): distal-less homeobox 5 (DLX5), 5'-GGC CGC TTT ACA GAG AAG GT-3' and 5'-GGT GAC TGT GGC GAG TTA CA-3'; osteopontin (OPN), 5'-TGA GTT TGG CAG CTC AGA GG-3' and 5'-TCG TCG TCG TCA TCA TCG TC-3'; osterix (Osx), 5'-TCG CAT CTG AAA GCC CAC TT-3' and 5'-TTG GCT TCT TCT TCC CCG AC-3'); and runt-related transcription factor 2 (Runx2), 5'-AAC CAA GTG GCC AGG TTC AA-3' and 5'-TAC TGG GAT GAG GAA TGC GC-3'); and GAPDH, 5'-TGG ATG GTC CCT CTG GAA-3' and 5'-GTG AGC TTC CCG TTC AGC-3'.

\section{Matrigel-based three-dimensional (3D) cultures}

The rDFSCs infected with Ad-GFP were collected and kept on ice and then resuspended in 200 $\mu \mathrm{L}$ cold Matrigel. The Matrigel-cell mixture was transferred to the middle of 24-well plates that had been preheated to $37^{\circ} \mathrm{C}$. The plates were then incubated at $37^{\circ} \mathrm{C}$ for $10 \mathrm{~min}$, followed by addition of $2 \mathrm{~mL}$ complete a-MEM medium. Cells were cultured in a humidified $37^{\circ} \mathrm{C}$ incubator with $5 \% \mathrm{CO}_{2}$. Fluorescence signals were examined by fluorescence microscopy.

\section{Cell proliferation assay}

The 96-well plates were coated with Matrigel and blank dishes were used as controls. The rDFSCs were seeded into 96 -well plates (1,000 cells/well) and then cultured in complete a-MEM medium for 1, 2, 3, 4,5 , and 6 days. At the indicated intervals, $20 \mu \mathrm{L}$ of Cell Counting Kit-8 (Beyotime) solution was added to each well and incubated for $2 \mathrm{~h}$ at $37^{\circ} \mathrm{C}$. The absorbance was detected at $450 \mathrm{~nm}$ using the PerkinElmer EnSpire ${ }^{\circledR}$ Multimode Plate Reader (PerkinElmer, San Jose, CA, USA).

\section{Ectopic bone formation assay}

The animal experiments were approved by the Ethics Committee of Chongqing Medicine University. All experimental procedures were carried out in accordance with the approved guidelines. Subconfluent rDFSCs were infected with Ad-BMP9 or Ad-GFP for $24 \mathrm{~h}$ and then collected and resuspended in $100 \mu \mathrm{L}$ Matrigel (Corning ${ }^{\circledR}$, Corning, NY, USA) or in $100 \mu \mathrm{L}$ PBS (control group). These mixtures were then injected subcutaneously $\left(1 \times 10^{6}\right.$ cells/injection site $)$ into five female athymic nude mice (5-6 weeks old; Experimental Animal Center of Chongqing Medicine University). The animals were maintained in a biosafety barrier facility.

\section{Micro-computed tomography (micro-CT) analysis}

At 5 weeks after implantation, the athymic nude mice were sacrificed, and the injection sites were retrieved and fixed in $10 \%$ buffered formalin. To quantitate bone formation, micro-CT imaging was performed. The fixed tissues were scanned using the VivaCT 40 scanner (Scanco Medical, Brüttisellen Switzerland) at a voltage of $70 \mathrm{kV}$ and beam current of $114 \mu \mathrm{A}$. The images were analyzed using $\mu \mathrm{CT}$ V6.1 software (Scanco Medical).

\section{Histological evaluation, immunohistochemical staining and trichrome staining}

Following micro-CT imaging, the retrieved tissues were decalcified using $10 \%$ ethylenediamine tetraacetic acid for 4 weeks and then embedded in paraffin. Serial sections $(4 \mu \mathrm{m})$ were obtained from paraffin blocks using a microtome (RM2125 RTS; Leica Biosystems, Wetzlar, Germany). Sections were deparaffinized, rehydrated, followed by incubation in $0.3 \%$ hydrogen peroxide solution for $10 \mathrm{~min}$ at room temperature. Nonspecific binding sites were blocked with $10 \%$ bovine serum albumin (Sigma-Aldrich) for $20 \mathrm{~min}$. The sections were incubated with mouse anti-osteopontin monoclonal antibody (1:100; Santa Cruz Biotechnology, CA, USA) or mouse antiosteocalcin monoclonal antibody (1:100; Santa Cruz Biotechnology) at $4^{\circ} \mathrm{C}$ for overnight, and the secondary antibody (1:600; Santa Cruz Biotechnology) at $37 \mathrm{oC}$ for $1 \mathrm{~h}$. The samples were developed with DAB (Sigma). The sections were subjected to hematoxylin and eosin (H\&E), as well as Masson's trichrome staining as described previously [24]. 


\section{Statistical analysis}

All experiments were performed in triplicate and/or repeated three times and results from one experimental replicate are shown as representative data. For the quantitative experiments, data are expressed as the mean \pm standard deviation. Differences among groups were compared using one-way analysis of variance and Scheffe's multiple comparison test. A value of $p<0.05$ was considered statistically significant.

\section{Results}

\section{BMP9 induced osteogenic differentiation of rDFSCs}

To assess the effects of BMP9 on osteogenic differentiation of rDFSCs, cells were infected with Ad-BMP9 or Ad-GFP (Fig. 1A), ALP activity was quantitated, and histochemical staining was conducted on days 3-5 after infection. The results showed that BMP9 significantly induced ALP activity in rDFSCs compared with the GFP control group (Fig. 1B). These results were confirmed by ALP histochemical staining (Fig. 1C, D). We further examined the expression of genes involved in osteogenesis by qRT-PCR. The results showed that the expression of all four osteogenic marker genes (Dlx5, $O P N, O s x$, and Runx2) were upregulated on days 3 and 5 when induced by BMP9. Dlx5, Osx, and Runx2 remained elevated on day 3 and gradually decreased from day 3 to 5, whereas OPN expression continued to increase from day 3 to day 5 of BMP9 induction (Fig. $1 \mathrm{E})$. To examine the mineralization capacity of the rDFSCs in the presence of BMP9, calcium deposition in cells was assessed by Alizarin Red S staining on day 14 of culture, which showed that the staining intensity was higher in the BMP9-treated group than in the GFP control group (Fig. 1F). Taken together, these results demonstrated that BMP9 effectively induced osteogenic differentiation of rDFSCs in vitro.
A
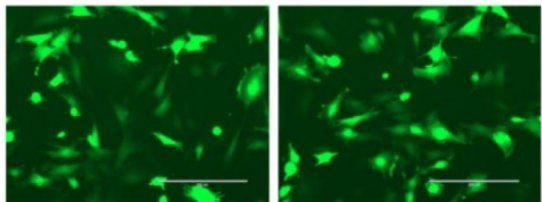

rDFSCs-GFP

rDFSCs-BMP9

$\mathrm{C}$

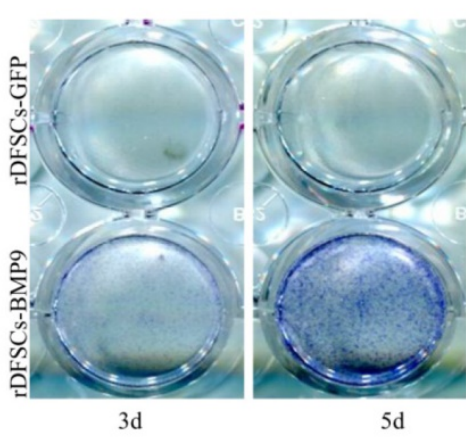

$\mathrm{E}$

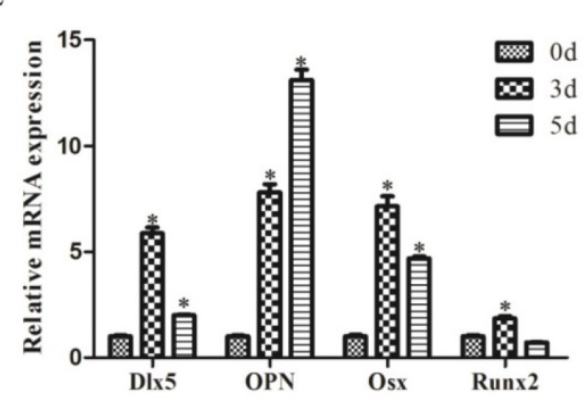

B

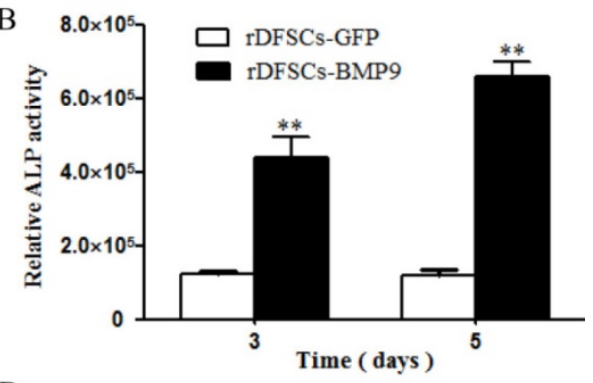

D

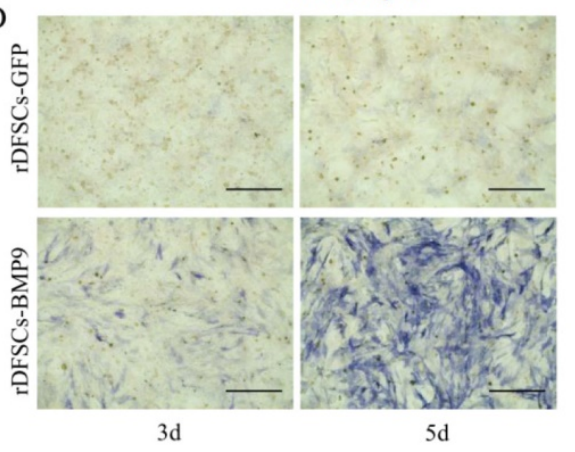

F

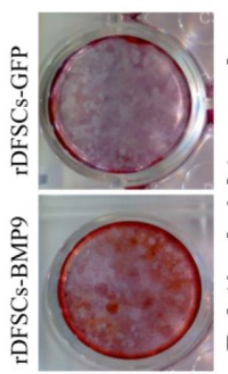

Figure 1. Bone morphogenetic protein (BMP) 9 promoted osteogenic differentiation of rDFSCs. A. Fluorescence images of rat dental follicle stem/precursor cells (rDFSCs) infected with adenoviruses-expressing human BMP9 or adenoviruses-expressing green fluorescent protein (GFP). B. A quantitative ALP activity assay conducted at the indicated time points. C. Total alkaline phosphatase (ALP) staining in the wells. D. Representative images of ALP staining of rDFSCs. E. Quantitative real-time polymerase chain reaction analysis of Dlx5, OPN, Osx, and Runx2 expression. F. Alizarin Red S staining of fixed rDFSCs at 14 days post-infection. More calcified nodules were observed in the BMP9 group compared with the GFP control group. Data are representative of three independent experiments. Scale bars represent $400 \mu \mathrm{m} .{ }^{*} \mathrm{p}<0.05$, ** $<<0.01$. 


\section{Cell spheroid growth in Matrigel 3D cultures in vitro}

To determine if Matrigel was compatible for growth and survival of rDFSCs and the potential use of Matrigel as an injectable thermoresponsive scaffold for rDFSC-based bone tissue engineering, we first evaluated in vitro cell growth in the presence of Matrigel. The rDFSCs exhibited spheroid growth at 7 days after mixing with Matrigel (Fig. 2A). The spheroid formation was thought to enhance multilineage differentiation potentials and upregulation of pluripotency marker genes in cells [25]. These results indicate that the Matrigel 3D cultures enhanced stemness in rDFSC spheroids. We then determined whether Matrigel affected the proliferation of rDFSCs in vitro. The cell growth curve showed that Matrigel did not affect the proliferation of rDFSCs (Fig. 2B).

\section{Matrigel enhanced BMP9-induced ectopic bone formation in rDFSC implantation in vivo}

A subcutaneous ectopic ossification animal model was used to evaluate the osteogenic potential of Matrigel for BMP9-transduced rDFSCs in vivo. Cells infected with Ad-BMP9 or Ad-GFP were mixed with Matrigel and injected subcutaneously into athymic nude mice. After 5 weeks, the mice were sacrificed, and the tissue masses that had formed at the injection sites were retrieved. Micro-CT analysis and 3D reconstruction were performed to evaluate new bone formation. Quantitative bone formation was determined by analyzing the micro-CT images. The rDFSCs transduced with GFP or mixed with Matrigel alone did not form any detectable masses (data not shown). Although there was no significant volumetric difference in the tissue masses of BMP9-transduced rDFSCs mixed with versus without Matrigel (Fig.
3A-C), the bone volume (BV) and bone surface (BS) were significantly larger in of the Matrigel group $(\mathrm{p}<0.01$; Fig. 3D, E). Immunohistochemical staining of osteocalcin $(O C N)$ and osteopontin $(O P N)$ revealed that osteoid matrix was present in the retrieved tissue masses (Fig. 3F). These results indicated that Matrigel effectively enhanced BMP9-induced osteogenic differentiation of rDFSCs.

\section{Matrigel enhanced BMP9-induced matrix mineralization in ectopic bones in vivo}

The subcutaneous tissue masses were retrieved, and paraffin sections were stained with H\&E. Histological analysis of the H\&E-stained sections indicated that BMP9-transduced rDFSCs formed trabecular structures only in the peripheral region of the masses and in the presence of a significant number of undifferentiated rDFSCs in the center. However, cells treated with both BMP9 and Matrigel led to more abundant and thicker trabecular structures distributed throughout the bone mass $(p<0.05$; Fig. $4 \mathrm{~A})$. Trichrome staining of the retrieved samples showed that BMP9-transduced rDFSCs delivered with Matrigel formed a more mature and fully mineralized bone matrix compared with BMP9- transduced rDFSCs injected directly $(p<0.05$; Fig. $4 \mathrm{~B})$. Together, these results indicated that the Matrigel scaffold promoted BMP9-induced osteoid matrix mineralization.

\section{Discussion}

Bone tissue engineering aims to induce functional bone regeneration and has been perceived as a better approach for the treatment of bone defects caused by fractures, nonunion, trauma, tumor resection, and other related diseases [26, 27]. Effective bone tissue engineering requires the synergistic combination of potent bone formation-stimulating factors, osteoblastic progenitors, and biocompatible scaffold materials. Among the osteo-
A

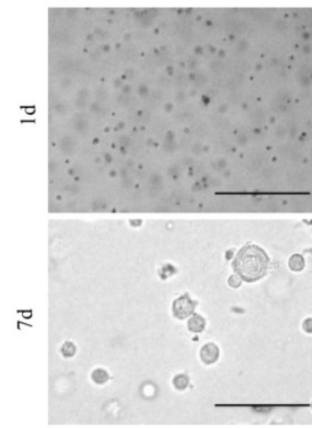

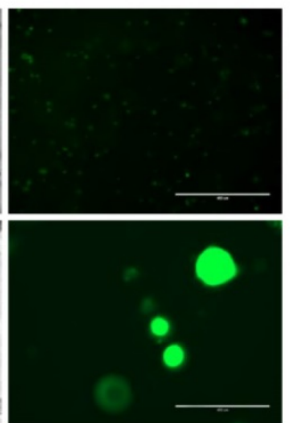

B

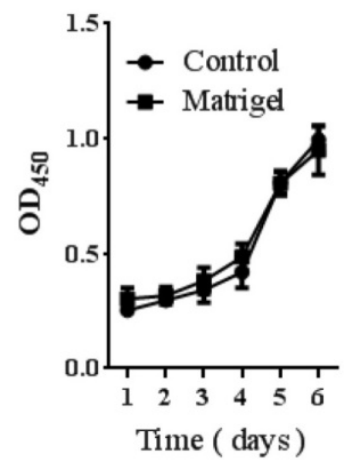

Figure 2. The effects of Matrigel three-dimensional (3D) cultures on rat dental follicle stem/precursor cell (rDFSC) spheroid formation. A. Representative images of rDFSC spheroids grown in Matrigel 3D scaffolds. Scale bars represent $400 \mu \mathrm{m}$. B. The 96 -well plates were coated with Matrigel and blank dishes were used as controls. The rDFSCs were seeded into 96-well plates and cultured for 1, 2, 3, 4, 5, and 6 days, followed by the Cell Counting Kit-8 proliferation assay. induction proteins, BMPs are considered one of the most potent osteoinductive factors. In this study, we demonstrated that overexpression of BMP9 remarkably promoted osteogenic differentiation in rDFSCs. Furthermore, mixing Matrigel with rDFSCs led to increased BMP9induced osteogenesis and matrix mineralization in ectopic bones.

At present, there are at least seven kinds of dental tissue-derived MSC-like populations that have been isolated and characterized, including dental pulp stem cells, stem cells from human exfoliated deciduous teeth, periodontal ligament stem cells, stem cells from 
apical papilla, DFSCs, gingival MSCs, and orofacial bone/bone marrow-derived MSCs [16, 28]. Dental tissue-derived stem cells are MSC-like progenitors that have a higher proliferation rate than that of bone marrow MSCs. For clinical applications, these dental stem cells are considered an attractive cell source for bone tissue engineering based on multiple advantages, such as easy access and preservation, and multilineage differentiation potentials [26, 28]. Compared with bone marrow MSCs and other dental stem cells, DFSCs are isolated more easily and grow readily under culture conditions. Moreover, DFSCs can be isolated from extracted impacted teeth that are normally discarded as medical waste during the course of dental treatment. These cells possess strong osteogenesis capability, which makes them an attractive cell source for repairing bone defects [29].

Previous studies reported that BMP2, 4, 6, 7, and 9 are osteogenic BMPs, and that BMP9 is the most effective member in inducing osteoblast differentiation of MSCs $[9,30]$. In the present study, BMP9 was overexpressed in rDFSCs by recombinant adenoviruses, and the osteogenic potency of cells was characterized by ALP expression (as an early marker), osteogenic marker gene expression, and matrix mineralization. The quantitative and qualitative ALP measurements showed that BMP9 significantly induced ALP activity in rDFSCs. The qRT-PCR results also indicated that the late osteogenic marker genes $D l x 5, O P N, O s x$, and Runx2 were significantly upregulated during BMP9 stimulation. The expression of these bone-related genes peaked around day 3 of Ad-BMP9 induction and then decreased at day 5. The data indicated that these genes had different roles at different time point of osteogenic differentiation, and they peaked at a certain induction time. Furthermore, Alizarin Red S staining showed the formation of calcium deposits, which were induced by BMP9. Together, these results indicated that BMP9 effectively promoted osteogenic differentiation in rDFSCs. It is worthy of note that BMP9 is reportedly to promote tumor growth, especially for bone tumors. Thus, BMP9 may be risky for certain bone defect repair procedures, such as osteosarcoma postoperative reconstruction [31].
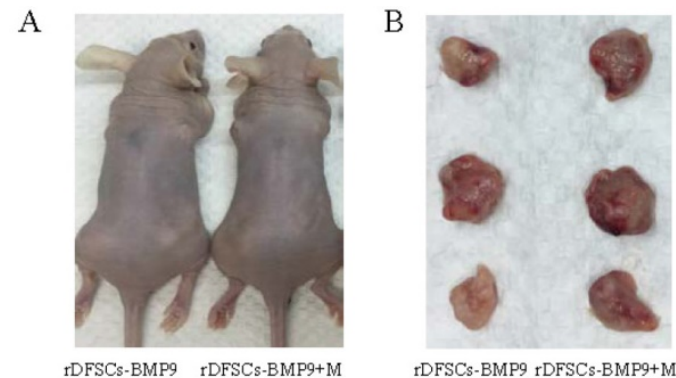

D

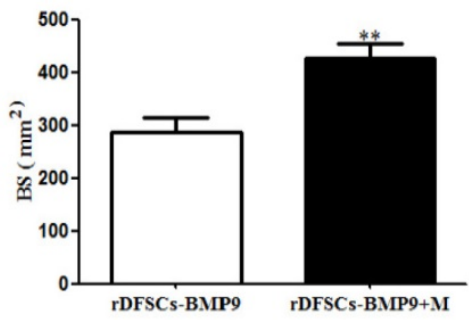

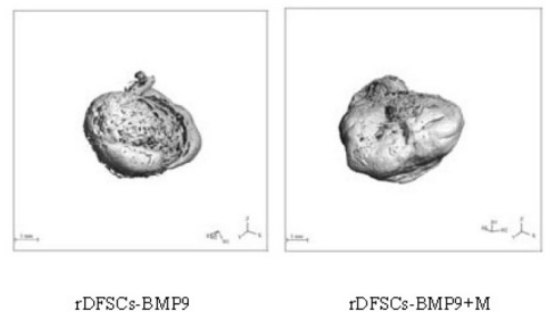

E

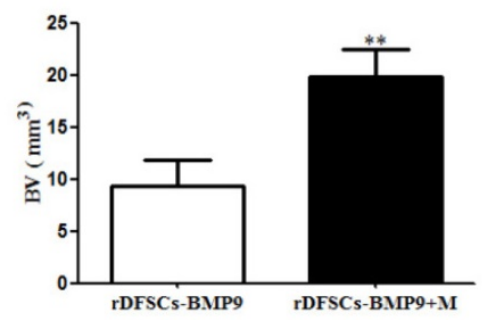

F

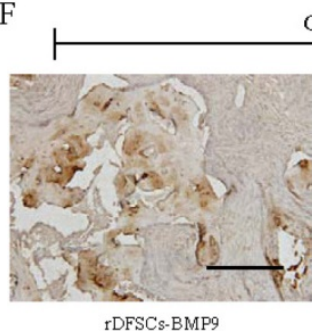

$\mathrm{OCN}$

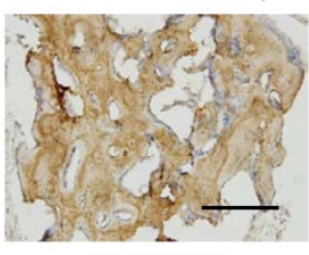

rDFSCs-BMP $9+\mathrm{M}$

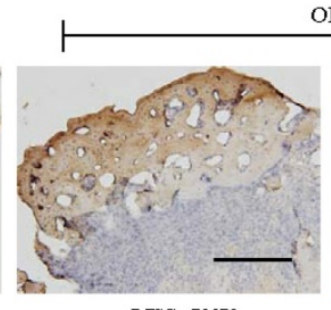

rDFSCs-BMP9
OPN

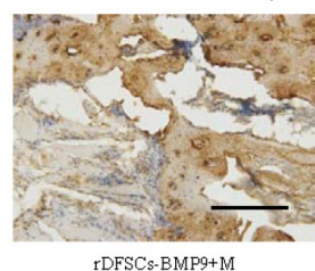

Figure 3. The effects of Matrigel on bone morphogenetic protein (BMP) 9-induced osteogenic differentiation of rat dental follicle stem/precursor cells (rDFSCs). A. Representative images of athymic nude mice injected subcutaneously with rDFSCs infected with BMP9 and mixed with Matrigel (rDFSCs-BMP9 + M) or without Matrigel (rDFSCs-BMP9). B. Representative images of tissue masses retrieved from the subcutaneous injection sites. C. Micro-computed tomography analysis and three-dimensional reconstruction of new bone formation in the two groups. D and E. Quantitative analyses of the (D) bone volume (BV) and (E) bone surface (BS) of new bones in the two groups. F. Immunohistochemical staining of tissue masses for osteo-markers. The positive images of $O C N$ and $O P N$ were observed in new bone tissue. Positive staining is labeled in brown. Scale bars represent $200 \mu \mathrm{m}$. ${ }^{* *} \mathrm{p}<0.01$. 
A

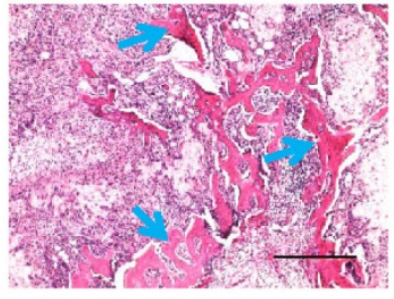

rDFSCs-BMP9

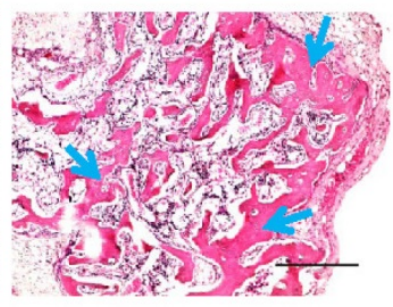

rDFSCs-BMP9+M

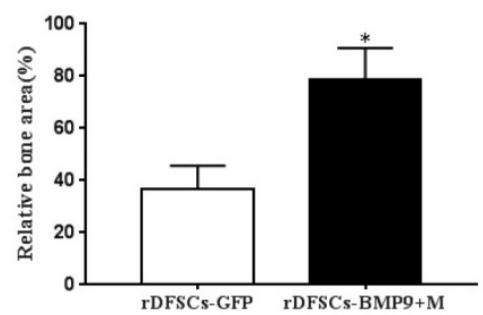

B

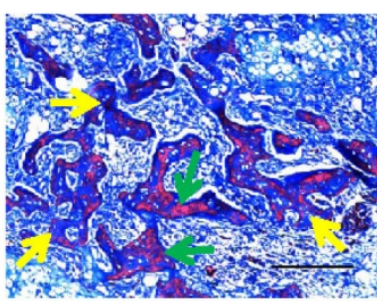

rDFSCs-BMP9

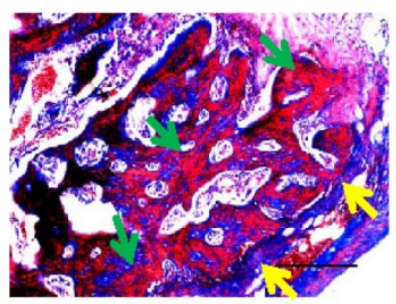

rDFSCs-BMP9+M

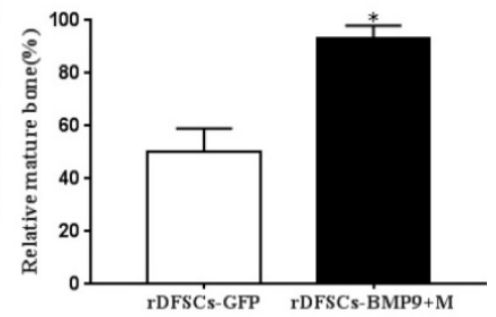

Figure 4. Histological examination of the retrieved bone masses. A. Hematoxylin and eosin staining of paraffin sections from ectopic bone. Blue arrows indicate osteoid matrix. Scale bars represent $400 \mu \mathrm{m} .{ }^{*} \mathrm{p}<0.05$. B. Representative images of trichrome staining of ectopic bone. Yellow arrows indicate unmineralized osteoid matrix. Green arrows indicate mineralized osteoid matrix. Scale bars represent $200 \mu \mathrm{m}$. ${ }^{*} \mathrm{p}<0.05$.

Scaffolding is one of three key components in tissue engineering-based strategies. The ideal scaffold for bone tissue engineering should be able to conform to the shape of the bone defect, easy to handle during surgery, and capable of promoting bone formation at the target site. Matrigel is a soluble and sterile extract of basement membrane proteins derived from Englebreth-Holm-Swarm tumors, which can form a 3D structure that is similar to extracellular matrix, to provide a more appropriate environment for tissue development or bone healing in vivo [32]. Matrigel contains growth and angiogenic factors, which can promote angiogenesis and prevent cells from undergoing apoptosis. Matrigel might also affect cell adhesion and proliferation and thus enhance the osteogenic potential of MSCs indirectly. Matrigel was superior to other matrices (Collagen I and Puramatrix) in terms of enhancing stem cell survival [33]. MSCs adhesion rate in 3D cultures containing Matrigel was much higher than that of the collagen cultures. Furthermore, MSCs grown in Matrigel showed competitive proliferative and osteogenic potential compared with those maintained in collagen gel [32]. Therefore, Matrigel might be considered to be a good hydrogel in bone regeneration. A previous study demonstrated that when mixed with gelatin, poly(polyethylene glycol citrate-co-N-isopropylacrylamide), a thermoresponsive biomacromolecule, was a suitable delivery vehicle for BMP9-transduced immortalized mouse embryonic fibroblasts and immortalized murine calvarial mesenchymal progenitor cells to promote localized bone formation and regenerate bone in murine critical-sized calvarial defects $[34,35]$. In the present study, we assessed whether Matrigel is a suitable delivery vehicle for BMP9-stimulated rDFSCs in improving bone formation. To directly observe the efficiency of transfection and infection of BMP9, green fluorescent protein (GFP) gene was incorporated into the adenoviral backbone. Transfected rDFSCs were monitored for GFP expression in our study. To determine whether Matrigel had good biocompatibility and was suitable for the growth and proliferation of rDFSCs, we performed the assessment of the in vitro growth of cells in the presence of Matrigel. We also found that rDFSC spheroids were formed in Matrigel 3D cultures, whereas their proliferation potential was not affected by the presence of Matrigel. Previous studies have reported that 3D spheroid cultures of human umbilical cord MSCs yielded more cells within the same volume, and the spheroids expressed higher levels of stem cell markers and displayed stronger multipotency [36]. Chen et al. reported that spheroid formation of human adipose-derived stem cells enhanced their properties of stemness, angiogenesis, and chemotaxis and thereby increased the regenerative potential of these cells for therapeutic uses [37]. Our in vivo study also showed that a Matrigel scaffold promoted the formation of bony masses in BMP9-induced rDFSCs. These results were further confirmed by histological examination of the retrieved bone masses, which showed that more mature and fully mineralized osteoid matrices were found in specimens treated 
with both BMP9 and Matrigel. Therefore, spheroid culturing of rDFSCs in Matrigel is one possible reason why Matrigel can accelerate BMP9-induced bone formation and osteoid matrix mineralization.

In summary, we demonstrated that BMP9 induced the osteogenic differentiation of rDFSCs, and that Matrigel facilitated BMP9-induced bone formation and matrix mineralization. These results suggest that Matrigel can be used as an injectable scaffold and delivery vehicle for BMP9-stimulated rDFSCs in bone tissue engineering.

\section{Acknowledgements}

This work was supported by the National Natural Science Foundation of China (81301551), the Chongqing Research Program of Basic Research and Frontier Technology (cstc2013jcyjA10022), the Visiting Scholar Foundation of Key Laboratory of Biorheological Science and Technology (Chongqing University), the Ministry of Education (CQKLBST-2012-004), the Scientific and Technological Research Program of Chongqing Municipal Education Commission (KJ170 2024), the Scientific and Technological Research Program of Chongqing Yubei district (2017 nongshe 42), and the Program for Innovation Team Building at Institutions of Higher Education in Chongqing in 2016 (CXTDG201602006).

\section{Ethical approval}

Ethical approval was approved by the Ethics Committee of Chongqing Medical University.

\section{Competing Interests}

The authors have declared that no competing interest exists.

\section{References}

1. Drago L, Toscano M, Bottagisio M. Recent Evidence on Bioactive Glass Antimicrobial and Antibiofilm Activity: A Mini-Review. Materials (Basel). 2018; 11.

2. Lee HG, Kim YD. Volumetric stability of autogenous bone graft with mandibular body bone: cone-beam computed tomography and three-dimensional reconstruction analysis. J Korean Assoc Oral Maxillofac Surg. 2015; 41: 232-9.

3. Ho-Shui-Ling A, Bolander J, Rustom LE, Johnson AW, Luyten FP, Picart C. Bone regeneration strategies: Engineered scaffolds, bioactive molecules and stem cells current stage and future perspectives. Biomaterials. 2018; 180: 143-62.

4. Giannoudis PV, Dinopoulos H, Tsiridis E. Bone substitutes: an update. Injury. 2005; 36 Suppl 3: S20-7.

5. Laurencin C, Khan Y, El-Amin SF. Bone graft substitutes. Expert Rev Med Devices. 2006; 3: 49-57.

6. Shi R, Huang Y, Ma C, Wu C, Tian W. Current advances for bone regeneration based on tissue engineering strategies. Front Med. 2018.

7. Langer R, Vacanti JP. Tissue engineering. Science. 1993; 260: 920-6.

8. Carreira AC, Lojudice FH, Halcsik E, Navarro RD, Sogayar MC, Granjeiro JM. Bone morphogenetic proteins: facts, challenges, and future perspectives. J Dent Res. 2014; 93: 335-45.

9. Kang Q, Song WX, Luo Q, Tang N, Luo J, Luo X, et al. A comprehensive analysis of the dual roles of BMPs in regulating adipogenic and osteogenic differentiation of mesenchymal progenitor cells. Stem Cells Dev. 2009; 18: 545-59.
10. Khosla S, Westendorf JJ, Oursler MJ. Building bone to reverse osteoporosis and repair fractures. J Clin Invest. 2008; 118: 421-8.

11. Luu HH, Song WX, Luo X, Manning D, Luo J, Deng ZL, et al. Distinct roles of bone morphogenetic proteins in osteogenic differentiation of mesenchymal stem cells. J Orthop Res. 2007; 25: 665-77.

12. Yan S, Zhang R, Ke W, Jing C, Huang S, Ji X, et al. Characterization of the essential role of Bone Morphogenetic Protein 9 (BMP9) in osteogenic differentiation of mesenchymal stem cells (MSCs) through RNA interference. Genes \& Diseases. 2018; 5: 172-84.

13. Cui J, Zhang W, Huang E, Wang J, Liao J, Li R, et al. BMP9-induced osteoblastic differentiation requires functional Notch signaling in mesenchymal stem cells. Laboratory Investigation. 2019; 99: 58-71.

14. Zhang H, Wang J, Deng F, Huang E, Yan Z, Wang Z, et al. Canonical Wnt signaling acts synergistically on BMP9-induced osteo/odontoblastic differentiation of stem cells of dental apical papilla (SCAPs). Biomaterials. 2015; 39: 145-54

15. Yao S, Pan F, Prpic V, Wise GE. Differentiation of stem cells in the dental follicle. J Dent Res. 2008; 87: 767-71.

16. Honda MJ, Imaizumi M, Tsuchiya S, Morsczeck C. Dental follicle stem cells and tissue engineering. J Oral Sci. 2010; 52: 541-52.

17. Mouriño V, Boccaccini AR. Bone tissue engineering therapeutics: controlled drug delivery in three-dimensional scaffolds. Journal of the Royal Society Interface. 2010; 7: 209.

18. Ramos-Hryb AB, Da-Costa MC, Trentin AG, Calloni GW. Matrigel supports neural, melanocytic and chondrogenic differentiation of trunk neural crest cells. Int J Dev Biol. 2013; 57: 885-90.

19. Kleinman HK, McGarvey ML, Liotta LA, Robey PG, Tryggvason K, Martin GR. Isolation and characterization of type IV procollagen, laminin, and heparan sulfate proteoglycan from the EHS sarcoma. Biochemistry. 1982; 21: 6188-93.

20. Jung YJ, Kim KC, Heo JY, Jing K, Lee KE, Hwang JS, et al. Induction of Angiogenesis by Matrigel Coating of VEGF-Loaded PEG/PCL-Based Hydrogel Scaffolds for hBMSC Transplantation. Moleculer Cells. 2015; 38: 663-8.

21. Park HJ, Lee WY, Jeong HY, Song H. Regeneration of Bovine Mammary Gland in Immunodeficient Mice by Transplantation of Bovine Mammary Epithelial Cells Mixed with Matrigel. International Journal of Stem Cells. 2016; 9: 186-91.

22. Nie L, Yang X, Duan L, Huang E, Pengfei Z, Luo W, et al. The healing of alveolar bone defects with novel bio-implants composed of Ad-BMP9-transfected rDFCs and CHA scaffolds. Sci Rep. 2017; 7: 6373.

23. Song $\mathrm{Y}$, Tang $\mathrm{Y}$, Song J, Lei M, Liang $\mathrm{P}, \mathrm{Fu} \mathrm{T}$, et al. Cyclic mechanical stretch enhances BMP9-induced osteogenic differentiation of mesenchymal stem cells. Int Orthop. 2018; 42: 947-55.

24. Huang E. Growth hormone synergizes with BMP9 in osteogenic differentiation by activating the JAK/STAT/IGF1 pathway in murine multilineage cells. Journal of Bone \& Mineral Research the Official Journal of the American Society for Bone \& Mineral Research. 2012; 27: 1566-75.

25. Cesarz Z, Tamama K. Spheroid Culture of Mesenchymal Stem Cells. Stem Cells Int. 2016; 2016: 9176357.

26. Amini AR, Laurencin CT, Nukavarapu SP. Bone tissue engineering: recent advances and challenges. Crit Rev Biomed Eng. 2012; 40: 363-408.

27. Black CR, Goriainov V, Gibbs D, Kanczler J, Tare RS, Oreffo RO. Bone Tissue Engineering. Curr Mol Biol Rep. 2015; 1: 132-40.

28. Mao X, Liu Y, Chen C, Shi S. Mesenchymal Stem Cells and Their Role in Dental Medicine. Dental Clinics of North America. 2017; 61: 161-72.

29. Rad MR, Bova JF, Orooji M, Pepping J, Qureshi A, Piero FD, et al. Evaluation of bone regeneration potential of dental follicle stem cells (DFSCs) for treatment of craniofacial defects. Cytotherapy. 2015; 17: 1572-81.

30. Cheng H, Jiang W, Phillips FM, Haydon RC, Peng Y, Zhou L, et al. Osteogenic activity of the fourteen types of human bone morphogenetic proteins (BMPs). J Bone Joint Surg Am. 2003; 85-A: 1544-52.

31. Liao J, Wei Q, Zou Y, Fan J, Song D, Cui J, et al. Notch Signaling Augments BMP9-Induced Bone Formation by Promoting the Osteogenesis-Angiogenesis Coupling Process in Mesenchymal Stem Cells (MSCs). Cellular Physiology \& Biochemistry. 2017; 41: 1905-23.

32. Kang BJ, Ryu HH, Park SS, Kim Y, Woo HM, Kim WH, et al. Effect of matrigel on the osteogenic potential of canine adipose tissue-derived mesenchymal stem cells. Journal of Veterinary Medical Science. 2012; 74: 827-36.

33. Cao F, Sadrzadeh Rafie AH, Abilez OJ, Wang H, Blundo JT, Pruitt B, et al. In vivo imaging and evaluation of different biomatrices for improvement of stem cell survival. Journal of Tissue Engineering \& Regenerative Medicine. 2010; 1: 465-8.

34. Dumanian ZP, Tollemar V, Ye J, Lu M, Zhu Y, Liao J, et al. Repair of critical sized cranial defects with BMP9-transduced calvarial cells delivered in a thermoresponsive scaffold. PLoS One. 2017; 12: e0172327. 
35. Ye J, Wang J, Zhu Y, Wei Q, Wang X, Yang J, et al. A thermoresponsive polydiolcitrate-gelatin scaffold and delivery system mediates effective bone formation from BMP9-transduced mesenchymal stem cells. Biomed Mater. 2016; 11: 025021.

36. Li Y, Guo G, Li L, Chen F, Bao J, Shi YJ, et al. Three-dimensional spheroid culture of human umbilical cord mesenchymal stem cells promotes cell yield and stemness maintenance. Cell Tissue Res. 2015; 360: 297-307.

37. Cheng NC, Chen SY, Li JR, Young TH. Short-term spheroid formation enhances the regenerative capacity of adipose-derived stem cells by promoting stemness, angiogenesis, and chemotaxis. Stem Cells Transl Med. 2013; 2: 584-94. 